\title{
Prognostic factors in primary breast carcinoma
}

\author{
S E Pinder, I O Ellis, C W Elston
}

The range of therapeutic options for the treatment of patients with primary breast cancer has widened considerably in recent years. The introduction of mammographic screening has led to the identification of greater numbers of small, stage 1 cancers. It is therefore increasingly important that clinicians are given the most accurate prognostic information on which to base the selection of the optimum therapy for each woman. In this way both under and over treatment can be avoided. This clinical need for information has resulted in an ever widening search for the ideal prognostic factor in primary breast cancer and consequently a multitude of papers have proposed numerous putative prognostic factors with differing degrees of significance. The current obsession with high technology has, to some extent, led to the value of traditional histopathology being overlooked, although the correlation between tumour morphology and degree of malignancy was first described over 100 years ago. This is unfortunate because routine histopathological examination of breast cancer specimens can yield a great deal of clinically useful information.

Histological grade provides prognostic information in many tumours, including breast cancers. Two main methods have evolved, based either on nuclear factors or a combination of cellular features (nuclear, cytological and architectural). The latter method is the most widely accepted for grading breast cancer and has been refined with the stricter definition of more objective criteria. ${ }^{1}$ As a result, the reproducibility of histological grading systems for breast carcinoma, previously questioned, has been improved; several recent studies have confirmed that good correlation between pathologists can be obtained if strict criteria are used. ${ }^{2-4}$

In the Nottingham breast cancer grading method the three features assessed are tubule formation, nuclear size/pleomorphism and mitotic count. Tumours in which less than $10 \%$ of the carcinoma is forming tubules score 3, those with 10 to $75 \%$ score 2 and if more than $75 \%$ of the cancer is forming central lumina, a score of 1 is given. Mild, moderate and high nuclear size/pleomorphism, when compared with normal epithelial cells score, 1, 2 and 3, respectively. For mitotic counts, a score is given according to the field area of the high power lens used. Only nuclei with clear features of metaphase, anaphase or telophase are included; apoptotic and hyperchromatic nuclei are discounted. Overall tumour grade is based on the final score of these three features. A score of 3,4 or 5 implies a grade 1 cancer, a score of 6 or 7 a grade 2 tumour and 8 or 9 a carcinoma of histological grade 3 . The system is easy to use and can be performed rapidly with experience. Histological grade, assessed in this way, is a strong indicator of patient survival. Patients with grade 1 carcinomas have an $85 \%$ chance of surviving for 10 years compared with less than $45 \%$ for patients with grade 3 tumours. This recognition of histological grade as an important prognostic factor has now led to methods being devised for assessing grade on cytological preparations, with the aim of obtaining prognostic information preoperatively. ${ }^{56}$

In our routine practice in Nottingham histological grade remains the factor of greatest importance in predicting prognosis for patients with primary operable breast carcinomas, with lymph node stage second in importance in multivariate analysis. All excised nodes should be examined histologically in a separate cassette. We advise that the blocks are taken by serial slicing perpendicular to the long axis of the node, thus providing the microscopist with a large area of lymph node and peripheral sinus in one section. One slice of node is examined microscopically for approximately each $5 \mathrm{~mm}$ of the maximum dimension of the lymph node. Lymph nodes containing any foci of carcinoma by this method are regarded as positive for staging purposes. Stage 1 tumours are lymph node negative, stage 2 tumours have three or less involved lymph nodes and stage 3 carcinomas have spread to four or more axillary and/or an internal mammary lymph node. The importance of micrometastases in prognosis remains unclear, partly because of differences in the definition of the size/volume and methods of assessing these lesions. Some groups have suggested that there is a prognostic association with disease free and overall survival for micrometastases identified by serial sectioning of lymph nodes, ${ }^{7}$ whereas others have found that micrometastases identified by immunohistochemical examination predict recurrence in ductal but not in lobular carcinomas. ${ }^{8}$ Although undoubtedly a small number of cases with micrometastases can be identified by immunohistochemical examination with anti-cytokeratin antibodies, we have found no prognostic significance in this feature and do
Correspondence to:
Dr C W Elston.

Accepted for publication

21 February 1995
Histopathology, The City Ho

Hucknall Road

Hottingham NG5 1PB 
not perform immunohistochemistry routinely..$^{9}$

The assessment of the size of a primary breast carcinoma also provides independent prognostic information. Tumour size is measured (to the nearest millimetre) in the fresh state by the pathologist after incision of the carcinoma in two vertical planes and this is then confirmed after fixation when sections are selected. For tumours detected by mammographic screening, and those with an associated extensive in situ component, macroscopic measurement of size may not be appropriate; in these cases size may be assessed microscopically, using the stage micrometer. ${ }^{10}$

While histological grade, lymph node stage and tumour size are well recognised features that predict prognosis in primary breast cancer, ${ }^{11}$ in multivariate analyses in our series of patients tumour type ${ }^{12}$ adds independent prognostic information, although the effects of this on survival are relatively small. Tumour types may be grouped into four classes based on prognosis. Tumours of excellent prognosis ( $>80 \% 10$ year survival) include tubular, invasive cribriform, mucinous, and tubulo-lobular carcinoma. Those with a good prognosis $(60-80 \% 10$ year survival) include tubular mixed, mixed ductal with special type and alveolar lobular carcinoma. Invasive papillary, classic lobular, medullary, and atypical medullary carcinomas have a moderate prognosis overall (50-60\% 10 year survival) and ductal (no special type), solid lobular, mixed ductal with lobular, and lobular mixed carcinomas have a poor prognosis ( $<50 \% 10$ year survival). However, in multivariate analysis, when these tumour type groups are included with grade, the latter has greater influence. Some of the "special types" of carcinoma show a consistent histological grade. Tubular carcinomas-for example, are almost invariably of grade 1 morphology. Other types, albeit rarely, demonstrate variable histological grade; pure mucinous carcinomas have an excellent prognosis overall, but those of histological grade 2 have a significantly worse prognosis (in the good rather than the excellent prognostic type group) than grade 1 lesions and these should be recognised and reported as such. In particular, although lobular carcinoma is usually of histological grade 2, rarer forms are found to be of histological grades 1 or 3 and these also have a significantly better or worse prognosis, respectively. We believe, therefore, that all histological types of breast carcinomas should be graded, without exception.

It is well recognised that the proportion of the different types of carcinomas seen in mammographic screening programmes differs from that of tumours presenting in symptomatic practice; tubular carcinomas-for example, comprise only $2 \%$ of symptomatic carcinomas but $20 \%$ of cancers detected by screening. Histological types of carcinoma may also express different biological markers; lobular carcinomas are more likely to be positive for oestrogen receptor (particularly those of alveolar subtype) and cathepsin $D$, but vimentin, p53 and c-erbB-2 oncogene protein are rarely expressed. ${ }^{1314}$ Lobular carcinomas may also show different patterns of metastatic disease to ductal/no special type tumours. ${ }^{15}$ Thus, although the importance of histological type in predicting survival is limited, typing of breast carcinomas may facilitate the better understanding of the biology of this disease.

The recognition of the presence or absence of vascular (blood vessel or lymphatic) invasion adds valuable prognostic information in predicting for survival but also, more importantly, for local recurrence in patients who have had breast conservation and for flap recurrence in those women who have undergone mastectomy. ${ }^{1617}$ Strict criteria must be adhered to for good reproducibility of reporting; an endothelial lining, identified on haematoxylin and eosin stained sections, should be present if definite vascular invasion is to be diagnosed.

Although each of the five traditional histological variables described above is of independent prognostic significance for survival in multivariate analysis, the predictive value for each individual patient is improved by a combination of factors in the form of a prognostic index. Lymph node stage, histological grade and tumour size have the greatest weight in multivariate analysis in the Nottingham/Tenovus series and have been combined to form the Nottingham prognostic index (NPI). Appropriate weighting from multivariate analysis has given the formula $(0.2 \times$ tumour size $(\mathrm{cm})+$ lymph node stage $(1-3)+$ histological grade (1-3)) for this prognostic index and the results have been confirmed in prospective studies ${ }^{1819}$ and in other centres. ${ }^{20}$ The higher the NPI score, the worse the prognosis. In this centre the NPI is used for selection of therapy for each patient rather than basing the choice of treatment on any single prognostic factor. Patients with a NPI score of 3.4 or less have an equivalent survival to aged matched controls (3\% annual mortality) and therefore receive no systemic adjuvant treatments. Women with a prognostic index score of greater than 5.4 have a $30 \%$ annual mortality and conversely may wish to receive more aggressive adjuvant therapy; the tumours in such patients are frequently oestrogen receptor negative so cytotoxic therapy may be more appropriate than hormone treatment. Those patients with a NPI score between 3.4 and 5.4 have an annual mortality of $7 \%$ and the choice of adjuvant treatment for these women depends on other variables such as hormone receptor status, menopausal status and axillary disease.

Many new antibodies raised against hormone and growth factor receptors and cell cycle markers have been described and are of significance in predicting for outcome in univariate analyses. To date, however, these have proved to be of limited significance in the majority of multivariate analyses when the traditional histological factors are carefully assessed and recorded. For example, oestrogen, progesterone and epidermal growth factor receptors and NCRC11 immunoreactivity, c-erbB2, p53, and helix pomatia lectin expression are outweighed in multivariate analysis for survival when histological grade and type, lymph node stage and tumour size are included. ${ }^{19}$ Indeed, 
even assessment of tumour ploidy and methods for determining the growth fraction of the tumour are not of independent significance in multivariate analysis when histological grade is available. We continue to assess the new markers and technologies, but it is likely that a molecular classification of breast cancer will only become commonplace when treatment strategies are based on specific genetic or biological events, comparable with the current use of hormone receptor assays to assess the suitability of a patient for hormone treatment. For the present, however, the traditional morphological factors of histological grade and type, lymph node stage, tumour size, and vascular invasion remain the most robust prognostic factors; they are relatively simple to assess and should form the basis of all routine histopathological reports in cases of breast cancer.

1 Elston CW, Ellis IO. Pathological prognostic factors in breast cancer. I. The value of histological grade in breas creast cancer. I. The value of histological grade in breast cancer: experience from a large study with

2 Dalton LW, Page DL, Dupont WD. Histologic grading of breast carcinoma. A reproducibility study. Cancer 1994; 73:2765-70.

3 Frierson HF, Wolber RA, Berean KW, Franquemont DW, Gaffey MJ, Boyd JC, et al. Interobserver reproducibility of the Nottingham modification of the Bloom and Richardson histologic grading system for infiltrating ductal carcinoma. Am f Clin Pathol 1995;103:195-9.

4 Robbins P, Pinder S, de Klerk N, Dawkins H, Harvey J, Sterrett G, et al. Histological grading of breast carcinomas. (in press).

5 Hunt CM, Ellis IO, Elston CW, Locker A, Pearson D, Blamey RW. Cytological grading of breast carcinoma-a feasible proposition? Cytopathology 1990;1:287-95.

6 Robinson IA, McKee G, Nicholson A, D'Arcy J, Jackson PA, Cook MG, et al. Prognostic value of cytological grading of fine-needle aspirates from breast carcinomas. Lance 1994;343:947-9.
7 International (Ludwig) Breast Cancer Study Group. Prognostic importance of occult axillary lymph node micro-
metastases from breast cancers. Lancet 1990;335:1565-8.

8 de Mascarel I, Bonichon F, Coindre JM, Trojani M. Prognostic significance of breast cancer axillary lymph node micrometastases assessed by two special techniques: reevaluation with longer follow-up. $\mathrm{Br} \mathcal{F}$ Cancer 1992;66: 523-7.

9 Galea MH, Athanassiou E, Bell J, Dilks B, Robertson JF, Elston CW, et al. Occult regional lymph node metastases from breast carcinoma: immunohistological detection with antibodies CAM 5.2 and NCRC-11. F Pathol 1991;165 $221-7$.

10 National Coordinating Committee for Breast Screening Pathology. Pathology reporting in breast cancer screening. Sheffield: NHSBSP Publications, 1995.

11 Page DL. Prognosis and breast cancer. Recognition of lethal and favorable prognostic types. Am ₹ Surg Pathol 1991; and favorabe

12 Ellis IO, Galea M, Broughton N, Locker A, Blamey RW, Elston CW. Pathological prognostic factors in breast cancer. II. Histological type. Relationship with survival in a large study with long-term follow-up. Histopathology 1992; 20:479-89.

13 Domagala W, Markiewski M, Kubiak R, Bartkowiak J, Osborn M. Immunohistochemical profile of invasive lobular carcinoma of the breast: predominantly vimentin and p53 protein negative, cathepsin $\mathrm{D}$ and oestrogen receptor positive. Virchows Arch A Pathol Anat Histopathol 1993; 423:497-502.

14 Porter PL, Garcia R, Moe R, Corwin DJ, Gown AM. CerbB-2 oncogene protein in in situ and invasive lobular erbB-2 oncogene protein in in situ and in

15 Lamovec J, Bracko M. Metastatic pattern of infiltrating lobular carcinoma of the breast: an autopsy study. $\mathcal{F}$ Surg Oncol 1991;48:28-33.

16 Pinder SE, Ellis IO, Galea M, O'Rouke S, Blamey RW, Elston CW. Pathological prognostic factors in breast cancer. III. Vascular invasion: relationship with recurrence and survival in a large study with long-term follow-up. Histopathology 1994;24:41-7.

17 O'Rourke S, Galea MH, Morgan D, Euhus D, Pinder S, Ellis IO, et al. Local recurrence after simple mastectomy. Br $¥$ Surg 1994;81:386-9.

18 Todd JH, Dowle C, Williams MR, Elston CW, Ellis IO, Hinton CP, et al. Confirmation of a prognostic index in pinton CP, et al. Confirmation of a prognostic index

19 Galea MH, Blamey RW, Elston CE, Ellis IO. The Nottingham Prognostic Index in primary breast cancer. Breast Cancer Res Treat 1992;22:207-19.

20 Brown JM, Benson EA, Jones M. Confirmation of a longterm prognostic index in breast cancer. Breast 1993;2 $144-7$. 